\title{
Variability in life-history and ecological traits is a buffer against extinction in mammals
}

\section{Manuela González-Suárez* and Eloy Revilla}

Department of Conservation Biology, Estación Biológica de Doñana (EBD-CSIC) Calle Américo Vespucio $s / n, 41092$, Sevilla, Spain

*Correspondence: E-mail: manuela. gonzalez@ebd.csic.es

\begin{abstract}
Anthropogenic degradation of the world's ecosystems is leading to a widespread and accelerating loss of biodiversity. However, not all species respond equally to existing threats, raising the question: what makes a species more vulnerable to extinction? We propose that higher intraspecific variability may reduce the risk of extinction, as different individuals and populations within a species may respond differently to occurring threats. Supporting this prediction, our results show that mammalian species with more variable adult body masses, litter sizes, sexual maturity ages and population densities are less vulnerable to extinction. Our findings reveal the role of local variation among populations, particularly of large mammals, as a buffering mechanism against extinction, and emphasise the importance of considering trait variation in comparative analyses and conservation management.
\end{abstract}

\section{Keywords}

Comparative studies, intraspecific variation, mammalia, PanTHERIA, Red List, taxonomically informed regression, vulnerability.

\section{INTRODUCTION}

The loss of biodiversity is currently so widespread and occurring at such accelerated rates that we may be entering the Earth's sixth mass-extinction event (Barnosky et al. 2011). Anthropogenic degradation of the world's ecosystems is the main driver of this extinction process. However, existing threats do not affect all species or ecosystems equally. Instead, some species show rapid declines, whereas others are able to persist and even expand in the face of perturbation. Understanding why some species are more vulnerable to extinction than others is critical to improve conservation actions and to gain insight into how species interact with their environment (while responding to threats). A fundamental approach to address this question has been to explore the links between vulnerability and intrinsic species' traits. Diverse comparative studies have collectively shed light on the principles underlying vulnerability in a wide variety of species, from plants to mammals (Foufopoulos \& Ives 1999; Bielby et al. 2008; Cardillo et al. 2008; Mattila et al. 2008; Montoya et al. 2008; Okes et al. 2008; Sodhi et al. 2008; Davidson et al. 2009; Giam et al. 2011; Murray et al. 2011; Pocock 2011). In mammalian species in particular, several life-history (e.g. body mass and gestation length), ecological (e.g., population density) and behavioural traits (e.g., mating strategies) are clearly linked to risk of extinction (Purvis et al. 2000; Brashares 2003; Davidson et al. 2009; Fritz et al. 2009). In addition, multiple studies have shown that geographical range area is a strong correlate of extinction risk in diverse taxa (Bielby et al. 2008; Harris \& Pimm 2008; Davidson et al. 2009; Giam et al. 2011; Murray et al. 2011). In fact, the IUCN (International Union for Conservation of Nature 2010) uses small geographical range as a criterion for listing species as threatened.

The size of the range area is not a species' trait per se, but rather reflects both the characteristics of a species (e.g. dispersal ability, habitat specialisation) and the extrinsic factors that affect its distribution (e.g. habitat fragmentation, historical and geographical constraints). Species that occupy large range areas are less vulnerable because large ranges are often associated with large population sizes (Pimm 1988). In addition, large, diverse range areas are also associated with greater trait variability (Figures S1, S2 in the Supplementary Information). More variable species can occupy wider, more diverse areas; and at the same time, species in wider, more diverse ranges become locally adapted to different environments (or are more likely to change via genetic drift) and thus, exhibit greater trait variability. Previous research has suggested that species exhibiting higher trait variability or flexibility can invade more habitats (Sol \& Lefebvre 2000), establish more successfully in new areas (Forsman et al. 2012) and the fossil record shows that greater morphological trait variation provided a buffer against extinction in the past (Liow 2007; Kolbe et al. 2011). In addition, theoretical studies have shown that individual variability can influence population dynamics (Bolnick et al. 2011; González-Suárez et al. 2011), although whether greater trait variability increases or reduces risk of extinction appears to be model and context dependent (Filin \& Ovadia 2007).

Here, we use empirical data to test if interpopulation variability in several life-history, ecological and behavioural traits is associated with vulnerability to extinction in extant mammalian species. Past comparative analyses have shown that mean or median estimates of species' trait are associated with risk of extinction (Purvis et al. 2000; Brashares 2003; Davidson et al. 2009; Fritz et al. 2009); thus, we define regression models that include these known correlates of vulnerability (mean trait estimates) and add coefficients of variation (CVs) to evaluate if trait variability has some additional power to explain vulnerability. In addition, we investigate if any identified associations with $\mathrm{CV}$ reflect only an indirect effect of trait variability via its association with geographical range area, or if variability can act directly as a buffer against extinction (Fig. 1). For example, interpopulation variability may have a direct effect if there are distinct populations that respond differently to existing threats. Our results show that greater interpopulation variability in diverse traits is directly associated with reduced vulnerability to extinction, suggesting that intraspecific variation can act as a buffer against extinction in mammals. 


\section{Indirect effect only}

$\left.\left.\begin{array}{c}\text { Intrinsic trait } \\ \text { variability }\end{array}\right)+\begin{array}{c}\text { Geographic } \\ \text { range area }\end{array}\right) \begin{gathered}\text { Population } \\ \text { size }\end{gathered}$
RISK

Indirect and direct effects

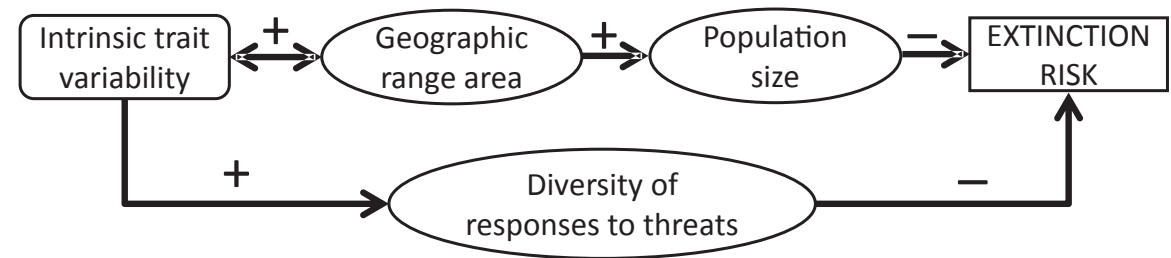

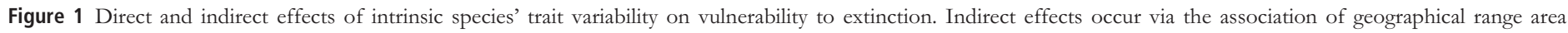

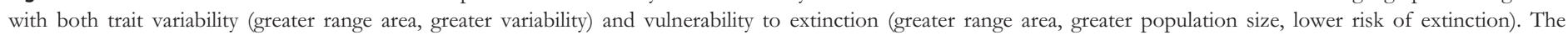

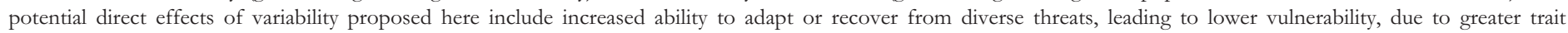
variability.

\section{MATERIALS AND METHODS}

\section{Databases}

To define mean and CV (interpopulation variability) trait values, we used the raw data file from the mammalian life-history data set PanTHERIA (Jones et al. 2009). The raw data file includes 99176 records representing estimates gathered from the literature for 25 traits describing morphology, development, reproduction, ecology and spatial data (Jones et al. 2009). Species names in the raw data file of the PanTHERIA database were tracked onto the Wilson \& Reeder's (2005) mammalian taxonomy based on the synonyms file provided by Jones et al. (2009). A total of 1349 records could not be tracked and were deleted, leaving a total of 97827 records describing 4204 mammalian species.

From the 25 traits available in the raw data files, we selected quantitative traits with available data for $>10 \%$ of species. From the remaining 15 traits, we excluded metabolic rate and maximum longevity and separated body mass into adult body mass and neonate body mass, resulting in 14 traits to be analysed (Table S1). For each trait, we defined the subgroup of mammals with at least two records per trait and then computed the arithmetic mean and the CV per species for each trait. CVs reflect variation among estimates reported by different studies (interpopulation variation), which measures individual variation within a species. Additional variation may be found among individuals within populations, but we had no available data to estimate this additional source of intraspecific individual variation (see Discussion). PanTHERIA records classified as minimum or maximum estimates were excluded prior to calculating mean and $\mathrm{CV}$. We detected a few errors in the original raw data file, which were corrected or deleted prior to calculating the final mean and CV estimates per species (Table S2).

As mentioned by Jones et al. (2009), PanTHERIA may contain duplicate entries which could affect our calculations, in particular of the CV. Duplicates could not be identified directly because the original source reference was not readily available for all entries. For traits measured as real numbers (e.g. body mass), Jones et al. (2009) describe a procedure to address this issue consisting in the removal of any repeated values. However, this duplicate removal approach cannot be extended to traits measured in integers (e.g. litter size), for which repeated values are less likely to indicate duplicates. Therefore, we initially used all data to estimate mean and $\mathrm{CV}$, but generated an alternative data set with only unique entries for traits measured in real numbers. Because results from both data sets were qualitatively the same, we report only those based on the complete data set.

Jones et al. (2009) calculated central tendency from the raw data files using estimated median values adjusted with offsets from generalised linear models that included different limiters (e.g. sex). This approach reduces the bias associated with treating distinct measures as equivalent (e.g. combining body mass of males and females in sexually dimorphic species), but it may overcorrect data. We found a very high correlation between Jones et al.'s median estimate and the arithmetic mean $(r>0.97)$ in all variables and thus, for this study we used the arithmetic mean. However, to be cautious, for traits with slightly lower correlations $(0.97<r<0.99$; population density, group size, gestation length, interbirth interval and weaning age), we compared results based on the arithmetic mean and the median. Overall, we found no qualitative differences, and only report results based on the arithmetic mean.

\section{Comparative analyses: trait variation and vulnerability}

For a first set of analyses, vulnerability was defined as the threat status from the 2008 Red List classification (International Union for Conservation of Nature 2010). We excluded Data Deficient species and grouped the Extinct and Extinct in the Wild categories. We also considered an alternative definition of vulnerability based on the global population trend categories defined by the IUCN. The IUCN requires that all species' assessments include an evaluation of the current global population trend classified into four categories: Increasing/Improving, Stable, Declining/Deteriorating and Unknown. To our knowledge, no quantitative criteria are provided to define trends. Trends classified as Unknown were excluded from the analyses. Both Red List status and population trend category were modelled as ordinal responses using a multinomial distribution with a cumulative logit link. Although the Red List classification has been traditionally converted into a continuous index for analyses (Purvis et al. 2000), this approach is unsatis- 
factory because the change in risk between categories (of Red List status or population trend) is not the same for each transition, as assumed by a continuous index. Therefore, we preferred to use a more realistic multinomial distribution. However, this limited our options for non-independence correction of analysis based on related species.

In comparative analyses, there is a potential issue of non-independence due to shared evolutionary history among species (Purvis 2008). Ideally, a well-defined phylogeny may be used to address this issue. However, although a good phylogeny exists for mammals (Bininda-Emonds et al. 2007), we are not aware of frequentist methods to implement phylogenetically corrected models with a multinomial distribution. Therefore, we used taxonomically informed generalised linear mixed models (GLMMs) to correct for non-independence (fitted in SAS 9.2, SAS Institute Inc., Cary, NC, USA). Models included nested random effects of order and family as defined by Wilson \& Reeder's taxonomy (2005). Including an effect of genus resulted in strong underdispersion, as data are often only available for one species per genus. Because taxonomic correction could be criticised as not fully correcting phylogenetic relationships, we also fitted all final models using phylogenetic generalised leastsquare models with Red List status and global population trend as continuous variables.

The high proportion of missing data in our data set made model selection and the potential use of aggregate measures (e.g. principal components) challenging because removing all missing data (complete case deletion) greatly reduced sample size and could bias estimates (Little \& Rubin 2002); yet, using imputation to fill data gaps is not appropriate because the missing data are prevalent and importantly, not missing at random (González-Suárez et al. in press). Imputation techniques are particularly not appropriate to fill gaps on trait variation because variation among populations is strongly context dependent and cannot be easily modelled as a function of evolutionary relationships or the values of other traits. Therefore, we developed an alternative approach to identify the most relevant traits while trying to maximise the use of all data available. First, we defined single-trait models for all 14 selected traits. Single-trait analyses explore if estimates of both mean and CV for each trait can explain vulnerability to extinction using all data available (for some species we only had data for a single trait, thus these species can only be considered in single-trait analyses). Second, we constructed multitrait models using a simplified data set that excluded traits with very little data (i.e. number of teats) and highly correlated traits $(r>|0.8|$ Table S3) to reduce multicollinearity. We also assess multicollinearity in the final models using Variance Inflation Factors (VIF). Among correlated traits, we excluded those with the smaller sample sizes: interbirth interval, and all morphological traits except adult body mass. Although population density and home range size are also highly correlated, both have similar sample sizes and thus, we ran separate analyses for each. The simplified data set includes nine traits.

To define a parsimonious multitrait model, we followed a threestep process (see appendix S1) that explores both linear and quadratic effects because previous studies have identified nonlinear relationships between species' traits and vulnerability to extinction (e.g. Cardillo et al. 2008). Our three-step process maximises the number of species considered by analysing increasingly complex trait combinations and thus, assesses the relevance of different traits in a more robust manner for the available information. Variable importance is determined via model AICs and variable weights evaluated at each step (Burnham \& Anderson 2002) with a last assessment of relevance based on the estimated slope coefficients in the final models. In particular, variables in the final model (already selected as important) are considered to be more strongly associated with vulnerability if the $90 \%$ confidence interval of the regression coefficient (calculated using the estimated standard error of the coefficient) does not overlap with zero. We used the $90 \%$ confidence interval as a conservative estimate of existing relationships following a neoFisherian approach (Hurlbert \& Lombardi 2009). In all steps, we included both mean and CV for each trait together. All trait variables were $\log _{10}$ transformed [for CV: $\left.\log _{10}(C V+1)\right]$. Previous studies have found that factors explaining vulnerability to extinction differ between large and small mammals (Cardillo et al. 2005; Davidson et al. 2009), indicating both groups should be explored independently. We ran separate multitrait analyses for large and small mammals using the same cutting point of $3 \mathrm{~kg}$ in mean adult body mass used by Cardillo et al. (2005).

There are few tools available to assess model fit in mixed models with a multinomial distribution, thus we used a simple classification approach to explore how well predictions matched observations. For each species, we determined the predicted status or trend as the level with highest probability, and compared this prediction to the listed Red List status or population trend to calculate prediction errors. Because in some cases several status levels had very similar probabilities, we also grouped probabilities and classified status into two categories: threatened $(\mathrm{T}=\mathrm{VU}+\mathrm{EN}+\mathrm{CR})$ and non-threatened $(\mathrm{NT}=\mathrm{LC}+\mathrm{NT})$. In this case, we used a 0.50 probability cutting point. This is a conservative assessment of model fit because predictions are based on a model that includes the observations for which predictions are made.

Finally, because the number of records available per species varies considerably, and $\mathrm{CV}$ values generally increase with sample size, we worried that estimated CVs did not reflect true trait variability, but rather could be an artefact of sample size. To explicitly control for this possibility, we included sample size as a continuous random factor in all analysed models. In addition, after running all analyses, we explored the relationship between CV of traits linked to vulnerability and the number of data entries per species. As a final test, we generated bootstrapped samples for each trait included in the final models and retested the relevance of CVs. For each of the five traits, we generated 200 samples per species of $n$ observations each (with replacement), where $n$ is the rounded mean number of available records per species (body mass $n=10$, litter size $n=12$, sexual maturity age $n=9$, population density $n=26$ and weaning age $n=7)$. Using this approach, we aimed to represent the 'mean sampled species'. Manly (2007) indicates that 100200 samples are an appropriate bootstrap sample to estimate standard deviation and hence CV. Using the new samples, we calculated 200 bootstrapped mean and CV estimates per species for each trait. These estimates were based on equal sample sizes $(n)$ for all species, although for species with few observations, the samples included mostly repeated records as no other information is available. Records for each trait are independent and thus, we had to generate separate bootstrapped samples for each trait, which complicated the use of the bootstrapped values to fit models (e.g. there would be $200 \times 200$ possible combinations of the bootstrapped samples for a model with just two traits). Our approach instead was to calculate an arithmetic mean of the 200 bootstrapped means 
and a mean, lower and upper 95\% confidence interval (CI) of the 200 bootstrapped CVs to test the robustness of our results with values based on equal-sized samples. We refitted the final models replacing the mean and $\mathrm{CV}$ of one trait a time for the bootstrapped mean (or $\mathrm{CI}$ ) and compared with the results based on the complete data set.

\section{Trait variation and geographical range area}

A challenge for comparative models that use Red List status to assess vulnerability is that testing if range area determines vulnerability is a circular argument for species listed as threatened because of their small range. The classical solution has been to exclude species listed as threatened under that criterion, but this biases the sample, as only threatened and small-range species (which exhibit lower trait variability) are excluded. Therefore, we took a different approach; first we focus on species' traits as described above, considering all species. Then for the analyses based on Red List status, we introduce geographical range area as a covariate analysing only the subset of species not listed under the small-range criterion. For analyses based on global population trend, we did not have to exclude any species after introducing range area because the definition of the trend categories is independent of geographical extent.

Geographical range area was estimated from the IUCN global distribution maps (International Union for Conservation of Nature 2010) using the cylindrical equal area projection and selecting only areas of the range defined as presence 'extant' or 'probably extant'. Estimates were obtained for 2697 species of the 2761 in our data set.
Area estimates were $\log _{10}$ transformed for analyses. For each measure of vulnerability (Red List status and global population trend) and each group of species (all mammals, small or large), we compared five models: trait means $+\mathrm{CVs}$ (the final model defined above); means + CVs + area; means + area; means $+\mathrm{CVs}+$ area $+\mathrm{CVs}$ area interactions; and mean $+\mathrm{CVs}$-area interactions. All models with $\Delta$ AIC $<2$ were considered supported.

\section{RESULTS}

We obtained mean and CV estimates for 2761 mammalian species, but missing data are prevalent in the data set $(18-96 \%$ missing cases per trait for the 2761 species with some data; 58-98\% missing out of the total 5415 extant mammalian species. Table S1). In fact, no single species has estimates for all 14 traits considered in our analyses. The 2761 species in the data set represent taxonomic diversity generally well, and include species from all 29 extant mammalian orders, 151 of 153 families, and 1000 out of the 1230 genera recognised by Wilson \& Reeder (2005). However, some groups are much better represented than others. While data are available for $>79 \%$ of the carnivore and ungulate (Artiodactyla and Perissodactyla) species, only $<37 \%$ of all rodents are included.

\section{Trait variation and vulnerability: Red List status}

Both single-trait and multitrait analyses identify mean and CV from diverse species traits as correlates of vulnerability to extinction measured as Red List status (Tables 1, S4). In particular, the final multi-

Table 1 Results of multitrait taxonomically informed GLMM exploring how species' traits predict Red List status and global population trend. Coefficient estimates are given for the arithmetic mean and the coefficient of variation (CV). We modelled the probability of higher vulnerability (an increase in Red List status or decreasing population trend). $N$ is the sample size (number of species). A dash $(-)$ indicates variables tested, but not included in the most final model. Coefficient values in brackets are estimates of quadratic effects $\left(\mathrm{x}^{2}\right)$. Variance inflation factors (VIF) were calculated to assess multicollinearity among variables in all models. VIF $>5$ are generally considered problematic

\begin{tabular}{|c|c|c|c|c|c|c|}
\hline \multirow[t]{2}{*}{ Traits } & \multicolumn{6}{|c|}{ Coefficient estimates (SE) } \\
\hline & Mean & $\mathrm{CV}$ & Mean & $\mathrm{CV}$ & Mean & $\mathrm{CV}$ \\
\hline Red List status & \multicolumn{2}{|c|}{ All data $(N=318)$} & \multicolumn{2}{|c|}{ Small mammals $(N=490)$} & \multicolumn{2}{|c|}{ Large mammals $(N=148)$} \\
\hline Adult body mass & $-0.1(0.26)$ & $-9.8(5.48)^{*}$ & $\begin{array}{c}5.2(1.50)^{*} \\
{\left[-1.0(0.33)^{*}\right]}\end{array}$ & $-3.0(4.57)$ & - & - \\
\hline Population density & $-0.5(0.21)^{*}$ & $2.5(1.82)$ & - & - & $-0.7(0.21)^{*}$ & $\begin{array}{l}22.7(7.64)^{*} \\
{\left[-35.3(13.05)^{*}\right]}\end{array}$ \\
\hline Litter size & - & - & $-1.4(0.72)^{*}$ & $-7.4(3.95)^{*}$ & - & - \\
\hline Weaning age & - & - & $0.8(0.60)$ & $-2.6(2.31)$ & - & - \\
\hline Sexual mat age & $3.3(0.72)^{*}$ & $-0.9(3.14)$ & - & - & $4.1(0.79)^{*}$ & $-1.2(4.24)$ \\
\hline Classification error rate $e^{\dagger}$ & \multicolumn{2}{|c|}{$0.808(0.874)$} & \multicolumn{2}{|c|}{$0.841(0.904)$} & \multicolumn{2}{|c|}{$0.730(0.784)$} \\
\hline Variance inflation factors & \multicolumn{2}{|c|}{$<3.89$} & \multicolumn{2}{|c|}{$<1.38$} & \multicolumn{2}{|c|}{$<1.12$} \\
\hline Global population trend & \multicolumn{2}{|c|}{ All data $(N=470)$} & \multicolumn{2}{|c|}{ Small mammals $(N=265)$} & \multicolumn{2}{|c|}{ Large mammals $(N=245)$} \\
\hline Adult body mass & $\begin{array}{c}1.0(0.33)^{*} \\
{\left[-0.2(0.05)^{*}\right]}\end{array}$ & $-6.5(2.57)^{*}$ & $0.3(0.21)$ & $0.2(4.33)$ & - & - \\
\hline Population density & - & - & - & - & - & - \\
\hline Litter size & - & - & $-0.5(0.59)$ & $-9.6(4.41)^{*}$ & - & - \\
\hline Weaning age & $1.1(0.41)^{*}$ & $-1.6(1.49)$ & $1.6(0.69)^{*}$ & $-1.9(2.41)$ & - & - \\
\hline Sexual mat age & $0.8(0.41)^{*}$ & $-4.8(1.60)^{*}$ & $0.5(0.53)$ & $-4.4(2.32)^{*}$ & $0.4(0.55)$ & $-6.4(2.78)^{*}$ \\
\hline Classification error rate ${ }^{\dagger}$ & \multicolumn{2}{|c|}{0.700} & \multicolumn{2}{|c|}{0.758} & \multicolumn{2}{|c|}{0.592} \\
\hline Variance inflation factors & \multicolumn{2}{|c|}{$<3.16$} & \multicolumn{2}{|c|}{$<2.45$} & \multicolumn{2}{|c|}{$<1.01$} \\
\hline
\end{tabular}

$* 90 \%$ confidence interval does not overlap with zero.

$\dagger$ Classification error rates in parenthesis are for reclassified status: Threatened vs. Non-Threatened. 
trait model shows that higher Red List status is associated with lower adult body mass variation, lower mean population density and higher mean sexual maturity ages (Table 1). The multitrait model is based on data from 318 species representing 19 of the 29 extant mammalian orders. Although diverse taxonomically, the majority of the 318 species are non-threatened, with 78\% listed as Least Concern. We found some support for an alternative model including litters per year (Table S5), but this model was based on just 101 species. Nevertheless, it is interesting that at least for these species, vulnerability may also be influenced by reproductive traits. Results of each step of the model selection process are in Tables S6-S11.

The final model predicts relatively high (0.43) probability of being threatened for species with body mass $\mathrm{CV}$ near 0, e.g. Chacoan peccary (Catagonus wagneri), whereas species with greater variation in body mass, e.g. $\mathrm{CV}=0.5$ for the red deer (Cervus elaphus), have a 0.12 probability of being threatened (Fig. 2a). The effect of mean population density on the probability of being threatened appears to be particularly strong at low population densities, i.e. $<10$ ind/ $\mathrm{km}^{-2}$ (Fig. 2c), but most studied species live above that threshold (Fig. 2d). Densities of $<0.01$ ind $/ \mathrm{km}^{-2}$, such as those observed in the polar bear (Ursus maritimus), are associated with a 0.70 probability of being threatened, but the probability is less than half that value for densities $>8$ ind $/ \mathrm{km}^{-2}$ (e.g. wild boar, Sus scrofa). Finally, the model predicts the largest changes in vulnerability associated with differences in mean sexual maturity age (Fig. 2e), ranging from a 0.92 probability of being threatened for a mammal with a maturity age of 11 years (Asian elephant, Elephas maximus) to a probability of 0.30 for a mammal maturing within a year. Most mammalian species
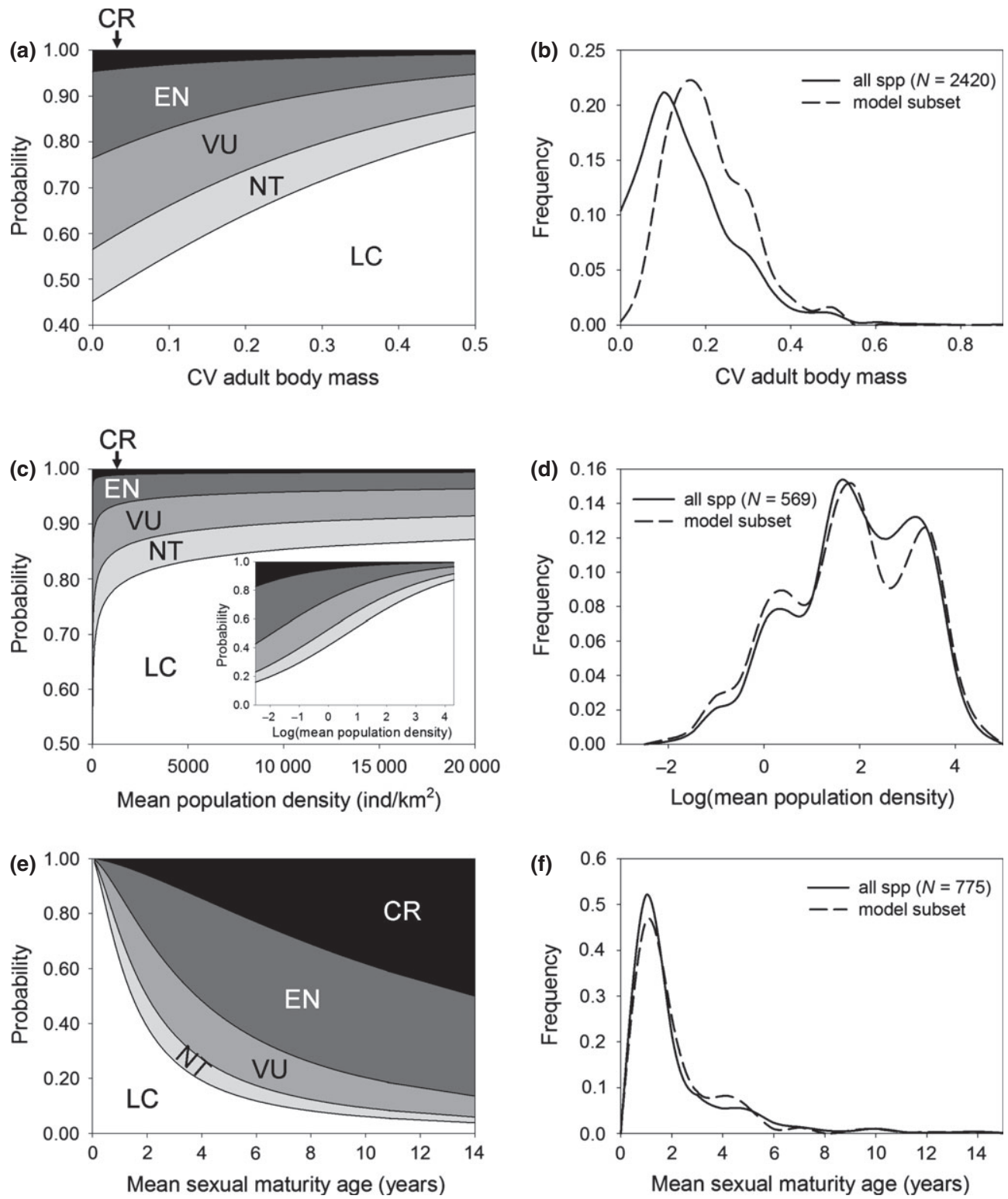

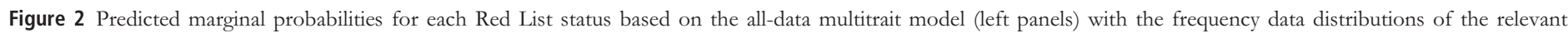

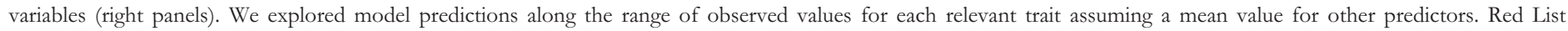

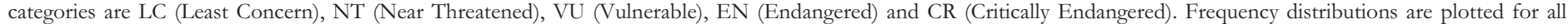

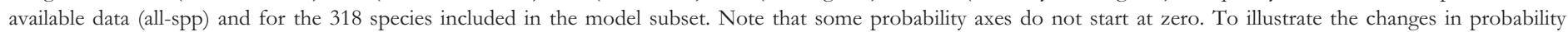
for mean population density better, we plotted predictions in the original scale and in a $\log _{10}$ scale (inset). 

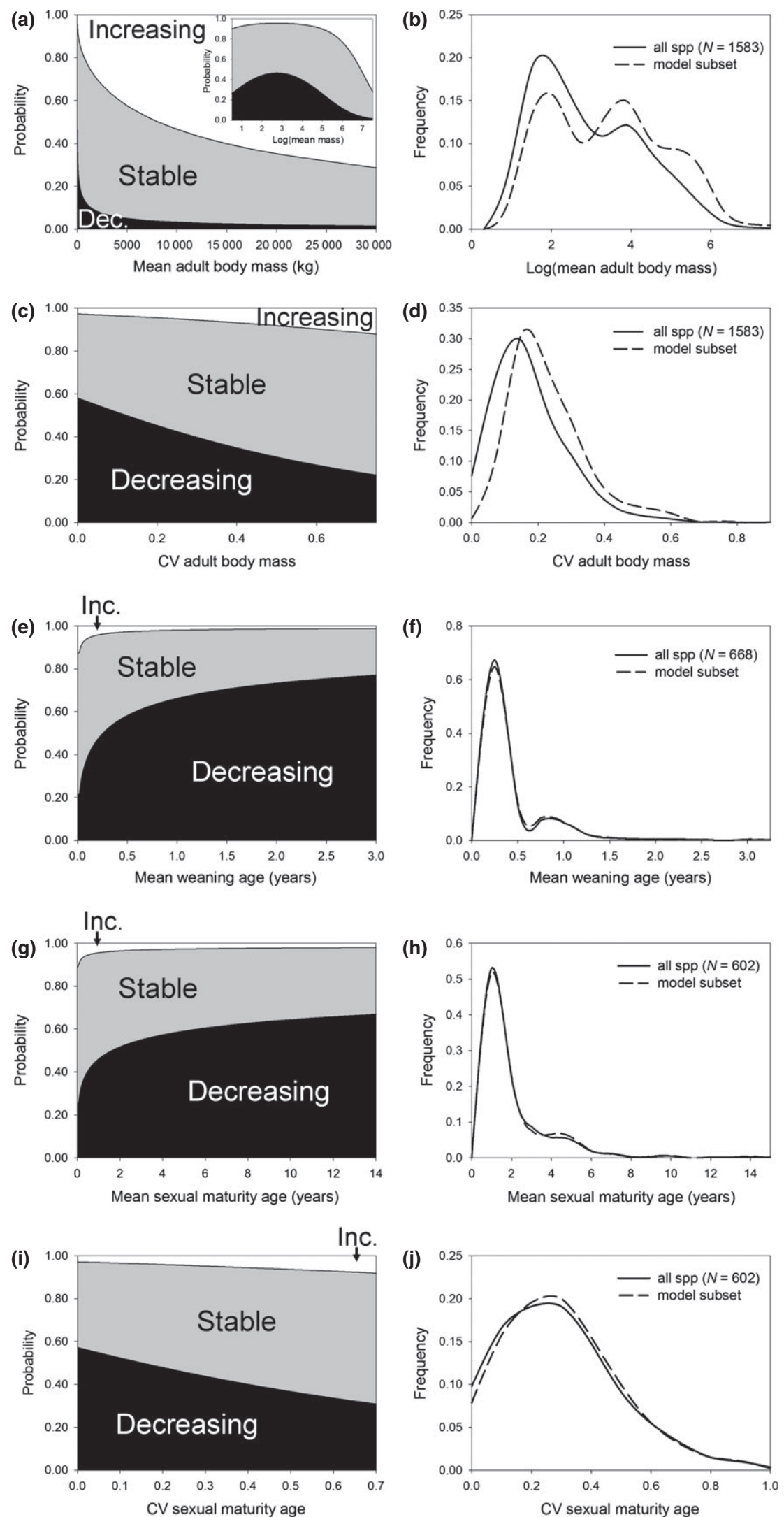

Figure 3 Predicted marginal probabilities for each global population trend category based on the all-data multitrait model (left panels) with the frequency data distributions of the relevant variables (right panels). We explored model predictions along the range of observed values for each relevant trait assuming a mean value for other predictors. Frequency distributions are plotted for all available data (all-spp) and for the 470 species included in the model subset. To illustrate the changes in probability for mean adult body mass better, we plotted predictions in the original scale and in a $\log _{10}$ scale (inset). 
with available data have sexual maturity ages $<4$ years (Fig. 2f), which correspond to probabilities of being threatened $<0.72$.

In both small and large mammals, interpopulation variation and mean trait estimates influence vulnerability to extinction (Table 1). Vulnerability to extinction appears to be more closely associated with reproductive and morphological traits in small mammals. In this group, higher Red List status is associated with intermediate values of mean adult body mass (c. $0.4 \mathrm{~kg}$ ), and lower mean and CV of litter size (Table 1). Meanwhile, for large mammals ( $>3 \mathrm{~kg}$ ), higher risk is associated with ecological and developmental traits including lower mean and intermediate CV (c. 1.1, quadratic effect) of population density, and higher mean sexual maturity age (Table 1$)$.

\section{Trait variation and vulnerability: global population trend}

Mean and CVs of intrinsic species traits are also associated with vulnerability to extinction measured as the IUCN global population trend categories (Tables 1, S4. Results of each step of the model selection process are in Tables S6-S11). In particular, the final multitrait model suggests that species with lower variation in adult body mass and in sexual maturity age, intermediate mean body masses and late mean ages for weaning and sexual maturation are more likely to have decreasing population trends (Table 1). The model is based on data from 470 species representing 22 of the 29 extant mammalian orders. Only $44(9.4 \%)$ of these species are listed as having an increasing population trend. This proportion, although small, is actually higher than expected based on all available data $(2.7 \%$ of 2983 mammals with defined global trend, excluding Unknown). The predicted effects of variation in both adult body mass and sexual maturity age are similar to each other (Figs 3c, i). Species with CV near 0 for these traits (e.g. Mediterranean monk seal, Monachus monachus) have c. 0.57 probability of a declining trend and $<0.03$ probability of an increasing trend. On the other hand, species with $\mathrm{CV}>0.7$ (e.g. stoat, Mustela erminea) have $<0.30$ probability of being declining and c. 0.10 probability of being increasing. Regarding mean trait estimates, the model predicts a very low probability of decreasing trend $(<0.11)$ for the larger mammals $(>1000 \mathrm{~kg})$, whereas species with mean body mass $c .0 .6 \mathrm{~kg}$ are the most likely to have declining population trends $(0.47$ probability of decreasing. Fig. 3a). Species with early weaning and maturity ages such as the field vole (Microtus agrestis, weaning at 2 weeks and maturing within 5 weeks) have a $<0.28$ probability of having a declining trend, whereas species weaning and maturing later, such as chimpanzees (Pan troglodytes, weaning after 2 years and maturing in 9 -10 years), have a probability $>0.63$.

Separate analyses of small and large mammals indicate similar effects of variation in sexual maturity age as the all-data results (Table 1). In addition, for small mammals, lower variation in litter size and higher mean weaning age are also associated with more negative population trends (Table 1). For large mammals, only variation in sexual maturity age is associated with vulnerability (Table 1). Results are qualitatively the same using a 0.6-kg cutting point to separate mammals into large and small (based on the alldata final model results), except that mean sexual maturity age is also a relevant variable for large mammals using this threshold.

Figure S3 shows generally weak relationships between relevant trait CVs and number of available observations, suggesting that CVs do not just reflect sample size. Furthermore, coefficient estimates based on bootstrapped mean and $\mathrm{CV}$ values are consistent with those estimated using the complete data set, indicating that our findings are not an artefact of unequal sample sizes (Table S12). Finally, our results are also robust to the approach used to correct phylogenetic non-independence. We obtained qualitatively similar results refitting the final models with phylogenetic generalised leastsquare models (Appendix S2, Table S13).

\section{Trait variation and geographical range area}

As expected, the fit of the final intrinsic trait models based on mean and CV for both Red List status and population trend is always improved by adding an estimate of geographical range area (Tables 2 and 3). However, models including CVs and interaction effects of range area and $\mathrm{CV}$ are supported $(\triangle \mathrm{AIC}<2)$ in nearly all analyses. Interaction terms generally indicate that the increase in vulnerability associated with small range areas is weaker for species with greater interpopulation variability. It is important to note that Red List models excluded species listed under criterion B. This correction did not simply change the number of species analysed, but biased the sample by excluding threatened species with small range areas and low CV estimates.

\section{DISCUSSION}

The mean and CV of diverse mammalian traits are associated with vulnerability to extinction measured either using Red List status or global population trend categories at least among mammalian species with available data. As in previous comparative studies (Cardillo et al. 2005, 2008), we find that which traits are actually identified as important depends on how vulnerability is assessed (Red List status or population trend) and which groups of species are considered (all or only small or large mammals). In general terms, threatened species can be characterised by a low number of individuals and little capacity to recover (with slow reproduction and long life cycles). Our results identify mean trait estimates associated with both general characteristics: higher risk for species living at low densities (generally implying fewer individuals) and higher risk for slowly reproducing species (late maturation and weaning ages, small litter sizes). In addition, we find that vulnerability is also associated with reduced interpopulation variation in diverse aspects of mammalian life history including development (sexual maturation age), morphology (body mass), reproduction (litter size) and ecology (population density). However, as discussed in the introduction, we must clarify if these associations simply reflect indirect effects via geographical range area or if there is evidence of direct effects (Fig. 1).

If variability only influences extinction risk indirectly via its association with range area, we would expect no support for models including CVs or interaction terms as the effect of variability on vulnerability would be effectively modelled using range. However, supported models include CVs and interaction terms in nearly all analyses, suggesting that variability can act directly as a buffer against extinction. Moreover, interaction terms indicate that species with greater trait variability are less vulnerable than expected based on the extent of their geographical range. This effect is particularly clear for large mammals (Tables 2 and 3). Greater trait flexibility may be more important as a buffer against extinction for large mammals because these species tend to have lower abundances, slower reproduction and long life cycles and thus, are more likely to be threatened by the reduced capacity to recover imposed by their 'mean' life history. 
Table 2 Results of taxonomically informed GLMM exploring how relevant species' traits, geographical range area and their interaction predict Red List status. Only supported models, $\triangle \mathrm{AIC}<2$, are shown. Coefficient estimates are given for the arithmetic mean and the coefficient of variation (CV) of traits included in the final models (Table 1), the range area and interactions. We modelled the probability of an increase in Red List status (higher vulnerability). $N$ is the sample size (number of species). Coefficient values in brackets are estimates of quadratic effects $\left(\mathrm{x}^{2}\right)$. A dash $(-)$ indicates a variable not included in the model

\begin{tabular}{|c|c|c|c|c|c|c|c|c|c|c|c|}
\hline Data & \multicolumn{6}{|l|}{ Traits } & \multicolumn{3}{|c|}{ Interactions of range with } & AIC & $\triangle \mathrm{AIC}$ \\
\hline \multicolumn{12}{|c|}{ All data $(N=307)$} \\
\hline \multirow[t]{2}{*}{ Range } & \multicolumn{2}{|l|}{ Mass } & \multicolumn{2}{|l|}{ Pop density } & \multicolumn{2}{|c|}{ Sex mat age } & \multirow[b]{2}{*}{$\mathrm{CV}$ mass } & \multirow[b]{2}{*}{ CV pop } & \multirow[b]{2}{*}{ CV sex mat } & \multirow[b]{3}{*}{331.9} & \multirow{4}{*}{$\begin{array}{l}0.00 \\
0.97\end{array}$} \\
\hline & Mean & $\mathrm{CV}$ & Mean & $\mathrm{CV}$ & Mean & $\mathrm{CV}$ & & & & & \\
\hline$-1.9(0.27)^{*}$ & $0.0(0.26)$ & - & $-1.0(0.22)^{*}$ & - & $2.9(0.76)^{*}$ & - & - & - & - & & \\
\hline$-1.2(0.61)^{*}$ & $0.1(0.29)$ & $-27.3(42.99)$ & $-1.1(0.23)^{*}$ & $-0.1(11.21)$ & $3.0(0.81)^{*}$ & $73.8(22.99)^{*}$ & $5.2(7.19)$ & $-0.1(1.84)$ & $-12.9(4.14)^{*}$ & 332.8 & \\
\hline \multicolumn{7}{|c|}{ Small mammals $(N=465)$} & & & & & \\
\hline \multirow[t]{2}{*}{ Range } & \multicolumn{2}{|l|}{ Mass } & \multicolumn{2}{|l|}{ Litter size } & \multicolumn{2}{|l|}{ Wean age } & & & & & \\
\hline & Mean & $\mathrm{CV}$ & Mean & $\mathrm{CV}$ & Mean & $\mathrm{CV}$ & & & & & \\
\hline$-1.2(0.22)^{*}$ & $\begin{array}{l}2.6(1.71) \\
{[-0.5(0.39)]}\end{array}$ & - & $-1.7(0.87)^{*}$ & - & $1.4(0.83)^{*}$ & - & & & & 364.3 & 0.00 \\
\hline \multicolumn{7}{|c|}{ Large mammals $(N=146)$} & & & & & \\
\hline \multirow[t]{2}{*}{ Range } & \multicolumn{2}{|l|}{ Pop density } & \multicolumn{2}{|l|}{ Sex mat age } & & & & & & & \\
\hline & Mean & $\mathrm{CV}$ & Mean & $\mathrm{CV}$ & & & CV pop & CV sex mat & & & \\
\hline$-1.9(0.33)^{*}$ & $-1.2(0.24)^{*}$ & $\begin{array}{l}17.8(7.87)^{*} \\
{\left[-30.5(13.22)^{*}\right]}\end{array}$ & $3.9(0.84)^{*}$ & $-1.0(4.30)$ & & & - & - & & 228.5 & 0.00 \\
\hline$-0.8(0.73)$ & $-1.3(0.24)^{*}$ & $\begin{array}{l}29.2(16.73)^{*} \\
{\left[-28.9(13.70)^{*}\right]}\end{array}$ & $4.2(0.88)^{*}$ & $45.4(33.20)$ & & & $-1.9(2.71)$ & $-8.2(5.80)$ & & 229.0 & 0.50 \\
\hline$-2.0(0.32)^{*}$ & $-1.2(0.23)^{*}$ & - & $3.7(0.81)^{*}$ & - & & & - & - & & 229.3 & 0.80 \\
\hline
\end{tabular}

$* 90 \%$ confidence interval does not overlap with zero.

Threatened species are broadly characterised by their low number of individuals and little capacity to recover. In addition, our results show that reduced trait variability (measured as interpopulation variation) is a third common attribute of threatened mammals. Interestingly, lower vulnerability is associated with greater variation in very diverse aspects of mammalian life history, suggesting multiple mechanisms by which variability may reduce extinction risk. Greater variability among populations may influence both the demographic effect that particular threats have (how threats reduce population size) as well as a species' ability to recover or adapt to new conditions. A separate possibility to explain our results is that low variability is a consequence, not a cause, of being vulnerable, as small populations in small ranges, which are vulnerable, are likely to have lower variability. While we cannot completely rule out that possibility because abundance data are not available, recent experimental work has shown that trait variation promotes establishment success and population growth (Forsman et al. 2012). In addition, our analyses show that correcting for range area low variability is still associated with vulnerability, indicating a direct effect. Future experimental work will be necessary to determine direct causality, but our study does show that low variability is a common attribute of threatened species. This finding emphasises the importance of considering trait variation in comparative analyses exploring risk of extinction, and importantly, in conservation management. If variation among populations can buffer a species against extinction, conservation efforts that focus on a single population may be less effective in protecting a species. In fact, the importance of preserving genetic variability is well recognised in conservation (Lande
1988; Lacy 1997), but because not all phenotypic variation is genetic, it is important to acknowledge the need to protect trait variability in a broader sense. Protecting diverse populations and ensuring that different individual phenotypes can coexist is a more general conservation goal, albeit perhaps not an easy one to accomplish.

Finally, we would like to note that in this study we used variation among populations to measure intraspecific variability, but that there is another potentially relevant source of variability: individual flexibility or variation within populations. Both sources ultimately reflect individual variation and may occur in one species, but are not necessarily correlated. In one extreme, different individuals may aggregate in distinct populations formed each by similar individuals (interpopulation variation without intrapopulation variation). Alternatively, distinct individuals may be uniformly distributed across populations (intrapopulation variation without interpopulation variation). In this study, we could not explicitly consider individual flexibility because available data do not include estimates of intrapopulation variability. However, it is likely that more flexible species in which individuals within a population can respond to changes in the environment by exhibiting or developing different morphology, behaviour or ecology are also less vulnerable to extinction (Rossmanith et al. 2006; Forsman \& Aberg 2008; Forsman et al. 2012), even if populations are relatively similar to each other. Future work would be necessary to assemble and analyse data sets that simultaneously capture intra- and interpopulation information and thus, address the role of both sources of individual flexibility as buffers against extinction. 


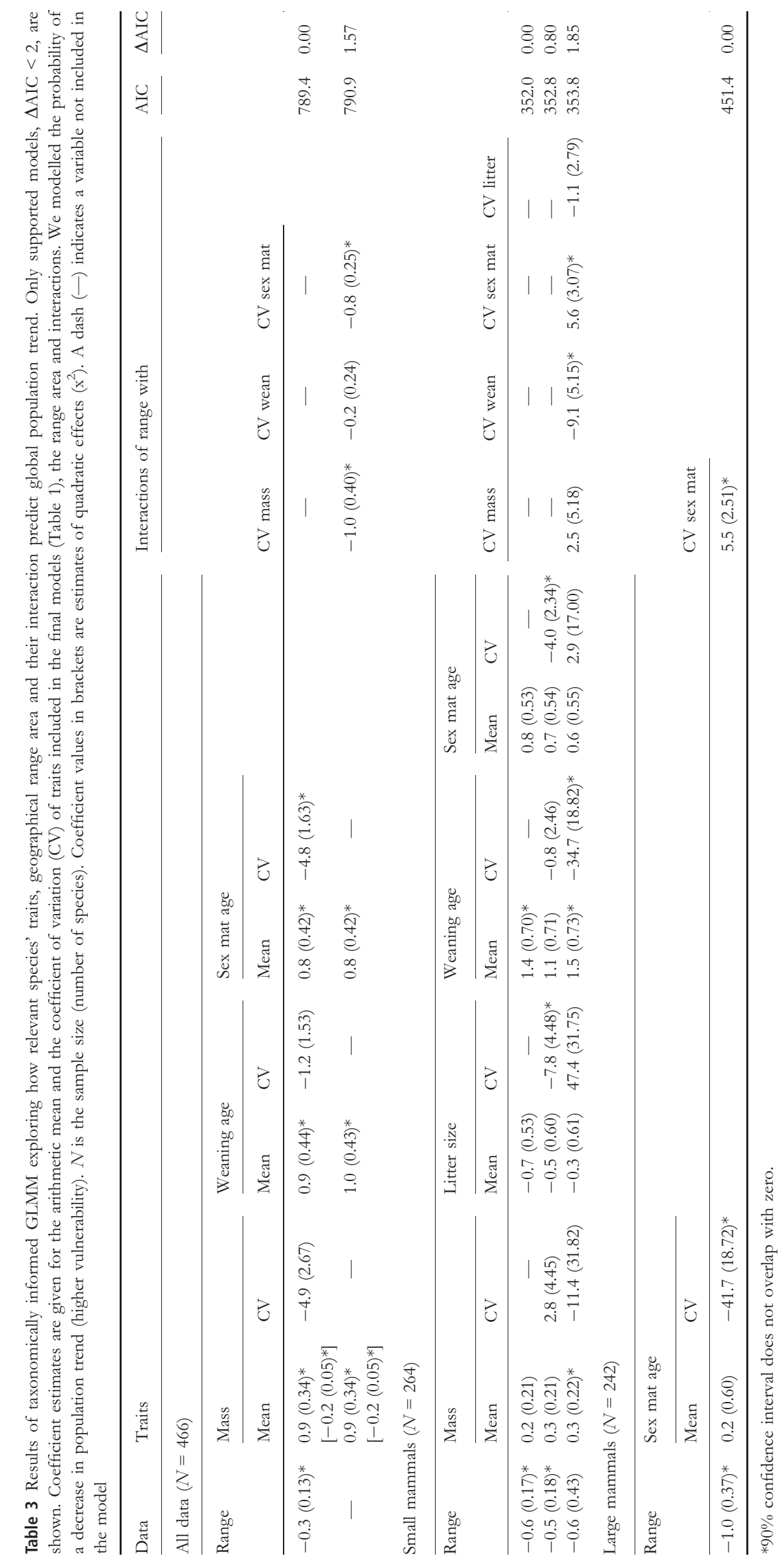




\section{ACKNOWLEDGEMENTS}

We thank Tim Coulson, nine anonymous referees and all CarNe members, particularly Miguel Clavero and Miguel Delibes, for comments on earlier versions of this manuscript. This work was funded by the European Community's Seventh Framework Programme (FP7/2007-2013) under grant agreement no. 235897, and the Spanish Ministry of Science and Innovation (CGL2009-07301/BOS, CGL2012-35931/BOS and JCI-2011-09158 cofunded by FEDER).

\section{AUTHORSHIP}

Both authors designed research and analysed results. MGS performed the analyses and wrote the paper with input from ER.

\section{REFERENCES}

Barnosky, A.D., Matzke, N., Tomiya, S., Wogan, G.O.U., Swartz, B., Quental, T. B. et al. (2011). Has the Earth's sixth mass extinction already arrived? Nature, 471, 51-57.

Bielby, J., Cooper, N., Cunningham, A.A., Garner, T.W.J. \& Purvis, A. (2008). Predicting susceptibility to future declines in the world's frogs. Conserv. Lett., 1, 82-90.

Bininda-Emonds, O.R.P., Cardillo, M., Jones, K.E., MacPhee, R.D.E., Beck, R. M.D., Grenyer, R. et al. (2007). The delayed rise of present-day mammals. Nature, 446, 507-512.

Bolnick, D.I., Amarasekare, P., Araújo, M.S., Bürger, R., Levine, J.M., Novak, M. et al. (2011). Why intraspecific trait variation matters in community ecology. Trends Ecol. Evol., 26, 183-192.

Brashares, J.S. (2003). Ecological, behavioral, and life-history correlates of mammal extinctions in West Africa. Conserv. Biol., 17, 733-743.

Burnham, K.P. \& Anderson, D.R. (2002). Model Selection and Multimodel Inference. A Practical Information-Theoretic Approach. Springer, New York, USA.

Cardillo, M., Mace, G.M., Jones, K.E., Bielby, J., Bininda-Emonds, O.R.P., Sechrest, W. et al. (2005). Multiple causes of high extinction risk in large mammal species. Science, 309, 1239-1241.

Cardillo, M., Mace, G.M., Gittleman, J.L., Jones, K.E., Bielby, J. \& Purvis, A. (2008). The predictability of extinction: biological and external correlates of decline in mammals. Proc. R. Soc. Lond. B Biol. Sci., 275, 1441-1448.

Davidson, A.D., Hamilton, M.J., Boyer, A.G., Brown, J.H. \& Ceballos, G. (2009). Multiple ecological pathways to extinction in mammals. Proc. Natl Acad. Sci. USA, 106, 10702-10705.

Filin, I. \& Ovadia, O. (2007). Individual size variation and population stability in a seasonal environment: a discrete-time model and its calibration using grasshoppers. Am. Nat., 170, 719-733.

Forsman, A. \& Aberg, B. (2008). Associations of variable coloration with niche breadth and conservation status among Australian reptiles. Ecology, 89, 12011207.

Forsman, A., Wennersten, L., Karlsson, M. \& Caesar, S. (2012). Variation in founder groups promotes establishment success in the wild. Proc. R. Soc. Lond. B Biol. Sci., 279, 2800-2806.

Foufopoulos, J. \& Ives, A.R. (1999). Reptile extinctions on land-bridge islands: life-history attributes and vulnerability to extinction. Am. Nat., 153, 1-25.

Fritz, S.A., Bininda-Emonds, O.R.P. \& Purvis, A. (2009). Geographical variation in predictors of mammalian extinction risk: big is bad, but only in the tropics. Ecol. Lett., 12, 538-549.

Giam, X., Ng, T.H., Lok, A.F.S.L. \& Ng, H.H. (2011). Local geographic range predicts freshwater fish extinctions in Singapore. J. Appl. Ecol., 48, 356-363.

González-Suárez, M., Galliard, J.-F.L. \& Claessen, D. (2011). Population and lifehistory consequences of within-cohort individual variation. Am. Nat., 178, $525-537$.

González-Suárez, M., Lucas, P.M. \& Revilla, E. (2012). Biases in comparative analyses of extinction risk: mind the gap. J. Anim. Ecol., 81, 1211-1222.

Harris, G. \& Pimm, S.L. (2008). Range size and extinction risk in forest birds. Conserv. Biol., 22, 163-171.
Hurlbert, S.H. \& Lombardi, C.M. (2009). Final collapse of the Neyman-Pearson decision theoretic framework and rise of the neoFisherian. Ann. Zool. Fenn., 46, 311-349.

International Union for Conservation of Nature (2010). IUCN Red List of Threatened Species. Version 2010.4. Available at: http://www.iucnredlist.org/. Last accessed 8 February 2010.

Jones, K.E., Bielby, J., Cardillo, M., Fritz, S.A., O’Dell, J., Orme, C.D.L. et al. (2009). PanTHERIA: a species-level database of life history, ecology, and geography of extant and recently extinct mammals. Ecology, 90, 2648-2648.

Kolbe, S.E., Lockwood, R. \& Hunt, G. (2011). Does morphological variation buffer against extinction? A test using veneroid bivalves from the PlioPleistocene of Florida. Paleobiology, 37, 355-368.

Lacy, R.C. (1997). Importance of genetic variation to the viability of mammalian populations. J. Mammal., 78, 320-335.

Lande, R. (1988). Genetics and demography in biological conservation. Science, 241, 1455-1460.

Liow, L.H. (2007). Does versatility as measured by geographic range, bathymetric range and morphological variability contribute to taxon longevity? Glob. Ecol. Biogeogr., 16, 117-128.

Little, R.J.A. \& Rubin, D.B. (2002). Statistical Analysis with Missing Data. Wiley, Hoboken, NJ.

Manly, B.F.J. (2007). Randomization, Bootstrap, and Monte Carlo Methods in Biology. Chapman \& Hall/CRC, Boca Raton, FL.

Mattila, M., Kotiaho, J.S., Kaitala, V. \& Komonen, A. (2008). The use of ecological traits in extinction risk assessments: a case study on geometrid moths. Biol. Conserv., 141, 2322-2328.

Montoya, D., Zavala, M.A., Rodríguez, M.A. \& Purves, D.W. (2008). Animal versus wind dispersal and the robustness of tree species to deforestation. Science, 320, 1502-1504.

Murray, K.A., Rosauer, D., McCallum, H. \& Skerratt, L.F. (2011). Integrating species traits with extrinsic threats: closing the gap between predicting and preventing species declines. Proc. R. Soc. Lond. B Biol. Sci., 278, 1515-1523.

Okes, N.C., Hockey, P.A.R. \& Cumming, G.S. (2008). Habitat use and life history as predictors of bird responses to habitat change. Conserv. Biol., 22, 151-162.

Pimm, S.L. (1988). On the risk of extinction. Am. Nat., 132, 757-785.

Pocock, M.J.O. (2011). Can traits predict species' vulnerability? A test with farmland passerines in two continents. Proc. R. Soc. Lond. B Biol. Sci., 278, 1532 $-1538$.

Purvis, A. (2008). Phylogenetic approaches to the study of extinction. Annu. Rev. Ecol. Syst., 39, 301-319.

Purvis, A., Gittleman, J.L., Cowlishaw, G. \& Mace, G.M. (2000). Predicting extinction risk in declining species. Proc. R. Soc. Lond. B Biol. Sci., 267, 1947-1952.

Rossmanith, E., Grimm, V., Blaum, N. \& Jeltsch, F. (2006). Behavioural flexibility in the mating system buffers population extinction: lessons from the lesser spotted woodpecker Picoides minor. J. Anim. Ecol., 75, 540-548.

Sodhi, N.S., Koh, L.P., Peh, K.S.H., Tan, H.T.W., Chazdon, R.L., Corlett, R.T. et al. (2008). Correlates of extinction proneness in tropical angiosperms. Divers. Distrib., 14, 1-10.

Sol, D. \& Lefebvre, L. (2000). Behavioural flexibility predicts invasion success in birds introduced to New Zealand. Oikos, 90, 599-605.

Wilson, D.E. \& Reeder, D.M. (2005). Mammal Species of the World: A Taxonomic and Geographic Reference. Johns Hopkins University Press, Baltimore.

\section{SUPPORTING INFORMATION}

Additional Supporting Information may be downloaded via the online version of this article at Wiley Online Library (www.ecologyletters.com). 